Revue internationale P.M.E.

Économie et gestion de la petite et moyenne entreprise

\title{
Continuité et reproductibilité des systèmes productifs territoriaux italiens
}

\section{Claude Courlet}

Volume 2, numéro 2-3, 1989

URI : https://id.erudit.org/iderudit/1007940ar

DOI : https://doi.org/10.7202/1007940ar

Aller au sommaire du numéro

Éditeur(s)

Presses de l'Université du Québec

ISSN

0776-5436 (imprimé)

1918-9699 (numérique)

Découvrir la revue

Citer cette note

Courlet, C. (1989). Continuité et reproductibilité des systèmes productifs territoriaux italiens. Revue internationale P.M.E., 2(2-3), 287-301.

https://doi.org/10.7202/1007940ar d'utilisation que vous pouvez consulter en ligne. 


\title{
Continuité et reproductibilité des systèmes productifs territoriaux italiens
}

\author{
Claude COURLET \\ Université des sciences sociales de Grenoble
}

\section{Introduction}

Avec la crise économique, de nouvelles localisations et de nouvelles structures de production émergent face aux territoires en difficulté. L'économie s'organise spatialement de façon à provoquer une dynamique industrielle émanant de nouvelles régions, de nouveaux espaces. On parle de comportements spatiaux nouveaux, d'un véritable retournement de hiérarchies spatiales (Aydalot, 1984) ou d'une «nouvelle centralité» des économies locales (Brutti, 1982).

Au centre de ces transformations, les petites et moyennes entreprises et, en particulier, les petites et moyennes industries. On sait que les structures spatiales ont été largement modelées par les grandes entreprises dominant les secteurs liés à la consommation de masse. Avec la crise, ce dynamisme majeur s'étiole. La régulation actuelle de celle-ci repose désormais sur les petites et moyennes entreprises industrielles. Ces PMI peuvent être des filiales de grandes entreprises relevant d'un calcul volontaire de «PMIsation» de la part de celles-ci. Mais ces PMI peuvent constituer aussi la partie d'un sous-système spécialisé dans la production de biens de petites et moyennes séries et donner lieu à un mode de développement original au niveau local. Celui-ci est souvent évoqué sous l'expression d'industrialisation diffuse.

- Maître de Conférence, Université des Sciences Sociales de Grenoble, docteur d'Etat en Sciences Economiques, spécialiste en économie du développement. Adresse : IREP Développement, B.P. 47X,38040 Grenoble Cedex (FRANCE) 
C'est sans doute en Italie, où existe une importante littérature à ce sujet, que ce phénomène connait le plus grand essor. Les études récentes sur l'industrie et l'économie régionale dans ce pays donnent une image de l'organisation économique du territoire tout à fait nouvelle par rapport aux analyses traditionnelles. A l'opposition, devenue classsique, entre le Nord industrialisé avec des grandes entreprises, et un Sud sous-développé et essentiellement agricole, se substitue désormais une réalité plus complexe.

Les zones de croisssance industrielle recoupent les régions italiennes du Nord-Est et du Centre (désignées souvent par le sigle NEC), soit 37 \% environ de la population nationale. Elles se caractérisent par la présence diffuse de petites entreprises donnant lieu à de véritables «zones systèmes» ou «systèmes productifs locaux» (Garofoli, 1983). G. Becattini parle - en reprenant l'expression d'Alfred Marshall - de districts industriels (Becattini, 1979).

Une véritable euphorie s'est emparée d'un certain nombre d'observateurs à propos de ce phénomène. En effet, l' intérêt actuel pour les districts industriels ou les systèmes territoriaux italiens tend vers le développement de vues par trop inconditionnelles de ces structures. A travers l'étude de celles-ci, il s'agit de plus en plus de l'analyse d'un modèle triomphant.

Ainsi, après l'attraction exercée par le fordisme, ou dans un autre domaine, par les industries industrialisantes, n'est-on pas en train de se laisser fasciner par les performances d'un nouveau modèle au risque de méconnaître ses véritables conditions de formation et de reproduction et donc, sa signification profonde?

Ce texte se propose d'apprécier la portée et les limites de ces types de structures à travers l'analyse de leurs origines, de leurs fonctionnements (2) et de l'examen des conditions de leur reproduction (3).

\section{Les systèmes productifs territoriaux en Italie : présentation du modèle}

\subsection{Origines et structuration des systèmes territoriaux}

\subsubsection{La ville comme trait d'union entre le système territorial et l'international}

Le d'́veloppement des systèmes territoriaux à industrialisation diffuse s'effectue dans un espace qui est typique de l'Italie des Communes (Fuà, 1985). Le territoire est constellé, de façon très dense, de villes moyennes et petites. Ce sont des centres aux fonctions urbaines diverses, avec des traditions d'administration locale et démocratique efficaces, jouissant d'une grande activité privée dans le commerce, 
les professions libérales et l'artisanat. Le territoire est desservi par un réseau routier dense. Chaque petite ville dispose de toutes les infrastructures sociales. Il n'y a pas de rupture entre la ville et la campagne : G. Becattini parle de «campagne urbanisée» (Becattini, 1979).

La ville joue le rôle fondamental de trait d'union entre le système local et les marchés internationaux. Cette fonction, qui remonte à l'époque des communes est essentielle car elle foumit l'information sur l'état des marchés extérieurs aux producteurs locaux. Depuis longtemps, ces villes sont le siège des marchands qui promeuvent et maintiennent les contacts avec le monde, ainsi que le siège des services relatifs à cette fonction.

Il en est de même pour la commercialisation de la production manufacturière. La ville est également le lieu où l'on vient apprendre les techniques de production. Aussi, depuis longtemps, le réseau de connaissances et de rapports urbains constitue-t-il une ressource importante pour la formation du milieu entrepreneurial garantissant en quelque sorte à chacun «une socialisation manufacturière», celle-ci n'ayant rien à voir avec la discipline tayloriste des usines situées dans les grandes villes.

\subsubsection{Le rôle de l'agriculture}

Certains espaces sont de formation récente : ceux-ci sont alors le produit de décentralisations productives avec prépondérance d'unités de production qui appartiennent à des entreprises externes. Mais beaucoup de systèmes territoriaux sont de formation ancienne et renvoient au rôle particulier de l'agriculture dans leur formation. Il s'agit de zones dont les origines remontent au 18ième/19ième siècle: Brescia, Biella, Lecco, Schio, Prato, Val Gandino, etc. Il s'agissait pour les paysans de ces régions de trouver un complément à leurs faibles revenus, d'où le développement d'une première activité industrielle. Ces zones ont ainsi connu ce que les historiens appellent une phase de proto-industrialisation (Dewerpe, 1984).

En particulier en raison d'une composante démographique tout à fait dynamique, ce lien étroit entre agriculture et industrialisation diffuse existe encore. On est en présence d'une structure spécifique de rapports de production dans l'agriculture avec prédominance des exploitations de petite dimension. Cette structure permet à l'industrialisation, à partir de petites initiatives, de s'appuyer sur une reproduction économique et sociale à dominante familiale et rurale (offre d'une main d'oeuvre flexible et à faible coût, organisation de la pluriactivité dans le cadre familial). Cette petite agriculture familiale est non seulement nécessaire à la reproduction de la force industrielle, elle est aussi le support des initiatives entrepreneuriales : mobilisation des terrains, des biens fonciers, pour créer l'entreprise par exemple. Ceci explique en grande partie l'intervention très fréquente de la famille dans la production, d'où l'existence des rapports de solidarité dans le cadre de la «famille élargie» et du voisinage. 


\subsection{Organisation et fonctionnement des systèmes territoriaux}

\subsubsection{Des champs spécifiques d'intervention}

L'industrialisation diffuse des systèmes territoriaux italiens s'oriente vers les secteurs, les technologies, les types de produits pour lesquels la petite dimension de l'entreprise n'est pas un obstacle. Dans un premier temps, il s'agit de produits à la portée de l'horizon culturel et des capacités techniques et financières des opérateurs locaux. Ceci implique - du moins dans les phases initiales de l'industrialisation - l'adoption de technologies pas trop éloignées de l'expérience d'un monde de petits agriculteurs et d'artisans. Elles sont en général caractérisées par une forte proportion de la main d'oeuvre dans la valeur ajoutée. Elles occupent souvent les créneaux laissés libres par la grande industrie (petit électroménager, petite mécanique, pièces diverses, etc.) ou ceux dont elles la délogent comme l'habillement et la chaussure. Elles peuvent évoluer - et sont encouragées en cela par le fractionnement croissant de la demande dans les pays industrialisés - vers des spécialisations de plus en plus poussées dans des productions de qualité et de petites et moyennes séries (certains vêtements, certaines fournitures mécaniques, électroniques, etc.) ou de mesure (machines spéciales, robots).

\subsubsection{Des systèmes intégrés de PME}

A la base de l'industrialisation diffuse, il y a la petite entreprise. Sa petite dimension la rend plus accessible à un promoteur local et plus adaptée aux choix d'une main d'oeuvre venant de la région ou du pays même. La préférence va vers des industries pour lesquelles il est possible de morceller le processus de production en phases ou en produits, sans avoir recours à des techniques dépassées. Ce morcellement de l'activité implique un réseau d'interdépendances entre les entreprises, de telle sorte que celles-ci participent à une véritable division du travail; il donne lieu à un système intégré de PME au sein duquel les entreprises entretiennent des liens qui vont de la participation financière (apparition de groupes locaux) au contrat de fourniture, au simple rapport de famille ou à l'accord informel temporaire. Cette division du travail renvoie à une fertilisation de l'économie formelle décentralisée : réseaux de PME et d'artisans, sous-traitance, travail à domicile. Elle concerne aussi l'utilisation de l'économie informelle : travail non déclaré, double emploi; heures supplémentaires non déclarées (Ritaine, 1987a).

\subsubsection{L'articulation productive}

Les structures productives ne peuvent être définies simplement à partir d'une base marchande et d'un découpage sectoriel débouchant sur la seule prise en considération des relations input-output. 
L'observation des faits conduit à mettre en évidence :

1) les liaisons (pas seulement marchandes) et synergies entre secteurs: métallurgie/ mécanique à Brescia; textile/vêtement à Bergame; bois/meubles en Vénétie; chaussures/industrie du caoutchouc/industrie chimique dans la province de Mantoue.

2) une complexification et diversification croissantes.

Les systèmes productifs locaux s'élargissent, conduisant à une diversification croissante. Mais celle-ci va dans le sens d'un renforcement des strucutres productives d'origine. Cela est la conséquence du développement des secteurs productifs étroitement liés au secteur de spécialisation d'origine. Ces nouvelles activités sont plus évoluées au niveau économique et technologique : taux de valeur ajoutée plus élevé, plus grande productivité du travail, intensité du capital plus grande, etc... Ceci conduit à une plus grande capacité d'innovation technologique et à un grand contrôle du marché (par exemple capacité de commercialisation). Cette diversification est aussi la conséquence du développement de secteurs qui fabriquent des machines, des instruments pour la production de biens typiques de l'espace.

Ainsi, autour de Milan :

- poche d'affinage, laboratoires pour doser le carbone et le gaz, calculateurs optimisant le fonctionnement des lances à ozygène et bien d'autres outils entourant les fours neufs des mini-aciéries (Brescia);

- machines pour la fabrication des chaussures (Vivegano);

- machines pour le textile-vêtement (Val Gandino);

- machines pour la production locale de matières premières et demi-produits de substitution : peaux synthétiques, fibres textiles et artificielles, etc. (Vivegano).

\subsubsection{Des conditions socio-économiques favorables}

Ce qui est remarquable, c'est l'existence d'une formation sociale au niveau territorial, suffisamment homogène du point de vue des comportements culturels et des aspirations : mobilité sociale élevée (de l'ouvrier au travailleur indépendant, au petit patron, etc.); structure culturelle qui favorise la mobilité sociale (gestion du temps de travail et du revenu au niveau de la cellule familiale, habitudes du calcul économique dans des zones de forte tradition paysanne, etc.)

Tout cela est médiatisé par des milieux professionnels, des structures familiales et communautaires, des rapports vicinaux. Les rapports de parentèles sont extrêmement denses et structurants du point de vue de l'économie. Les affaires se font souvent entre membres de familles alliées ou entre connaissances de longue date. La société locale peut faire ployer le fonctionnement du marché à ses propres règles (Bagnasco, 1985). La diffusion industrielle trouve alors une nouvelle ressource dans l'organisation collective, ressource de stabilisation et de reproduction (Ritaine, 1987a). Au total, on est en présence d' une économie et d' une société de réciprocité. 


\subsection{Flexibilité productive et économles d'agglomération}

L'homogénéité de la structure productive et sociale à l'intérieur d'un territoire restreint permet de garantir à la fois les avantages de la grande dimension, grâce aux économies d'agglomération et celui de la petite taille que constitue la flexibilité productive.

\subsubsection{La flexibilité productive}

Celle-ci est basée sur la petite dimension des unités de production, sur la densité des relations entre celles-ci et sur la rapidité de réponse des petites entreprises aux nouvelles conditions externes et internes de la zone. Par exemple à Prato, en quinze jours, on peut livrer un nouveau produit. Mais cette flexibilité, ce n'est pas seulement la capacité de répondre rapidement aux sollicitations externes du marché, c'est aussi la capacité d'adaptabilité aux nouvelles technologies. Dans ce domaine, petite taille et système intégré de PME semblent constituer un facteur tout à fait favorable (Bertrand, 1986). Dans la majorité des cas, l'évolution se fait progressivement dans une filière technique connue par une longue tradition. On voit le plus souvent l'ancien coexister avec le nouveau. L'adaptation ne paraît pas poser de problèmes : matières utilisées, machines textiles, machines outils. On voit aussi beaucoup de PMI se lancer dans des technologies entièrement nouvelles. Surtout, il semble y avoir convergence entre l'expansion rapide des petites entreprises fondées sur le principe de la flexibilité et l'adaptation rapide au marché et les possibilités offertes sur le plan technologique par une nouvelle génération d'automatismes.

Plus de souplesse et une meilleure disposition pour les petites innovations font aussi que le système des petites entreprises est mieux adapté à produire des articles en petite série ou en exemplaire unique. Il se trouve particulièrement adapté sur des marchés de plus en plus caractérisés par une plus grande fragmentation de la demande (par zone géographique ou groupes sociaux) et par une succession rapide des modes ou des innovations (Bertrand, 1986).

\subsubsection{Les économies d'agglomération}

Elles sont, quant à elles, la conséquence de rapports intenses entre les entreprises locales; ceux-ci amplifient la division du travail, permettant ainsi une spécialisation productive de plus en plus grande, l'introduction de nouvelles technologies et une plus grande efficacité du système local : baisse du coût unitaire de production et/ou augmentation de la productivité, possibilité d'élargir les débouchés aux niveaux national et international.

L'appareil productif local fonctionne ainsi comme un système unique d'interrelations entre entreprises et secteurs productifs, (infrasectorielles et intersectorielles). Les premières déterminent une spécialisation par phase productive dans le 
cadre d'un même secteur; les secondes augmentent l'intégration verticale dans le cadre du système productif local, le rendant ainsi de plus en plus complexe.

Cette toile serrée de relations confère aux systèmes territoriaux des économies d'agglomération qui se substituent en quelque sorte aux économies d'échelles typiques des grandes usines intégrées. Le rôle de ces économies d'agglomération semble d'autant plus important que le système productif local est plus développé. La contiguîté de nombreuses entreprises similaires procure à chacune d'elles un ensemble d'économies et de stimulants. Le processus d'agglomération une fois mis en route, un certain mouvement d'avalanche s'ensuit, soit par l'accroisssement des entreprises de l'extérieur, soit enfin par un effet de débordement ou d'annexion que la zone d'agglomération exerce sur les territoires environnants (Fuà, 1985).

Dernier facteur à signaler et qui contribue à la stabilisation des systèmes locaux : le système d'information qui se forme au niveau de l'espace concerné. Celuici garantit la circulation rapide des informations relatives aux débouchés, à la technologie, aux nouvelles matières premières, aux nouvelles techniques financières et commerciales.

Une fois encore, le cas de Patro illustre bien cette évolution avec la mise en place du système télématique inter-entreprises (SPRINT). Celui-ci vise à renforcer les avantages des petites entreprises en matière d'adaptabilité et de flexibilité, tout en surmontant le handicap que peut représenter le fractionnement du processus de production, avec tous les problèmes de circulation de l'information que cela représente. Ce système, à travers l'installation de 300 terminaux, doit permettre une meilleure communication et assurer la transparence du marché du travail et des plans de charge des sous-traitants. Ce programme réunit les entreprises locales, les banques, l'Eni, les télécommunications, les chambres de commerce et l'Université (Bertrand, 1986).

On peut aussi citer le cas du CITER à Carpi. Créé en 1980, ce centre d'études associe les entreprises et les associations patronales et artisanales du secteur textile. Il offre à ses associés une gamme de services d'information qui couvrent tous les domaines de la mode : tendance et évolution des couleurs, des matériaux, des lignes; un service d'information sur l'état et les tendances de la technologies; un service de recherche et de développement qui se concrétise en conférences, séminaires, publications, sur l'évolution du marché, les transformations socio-économiques, les techniques du marketing, etc. Au cours de ces évolutions, le système productif local franchit un pas important : il ne s'agit plus seulement de gestion d'entreprise, mais d'un réseau d'entreprises. 


\section{La reproduction des systèmes productifs territoriaux}

On vient de montrer que les systèmes territoriaux italiens constituent une forme de développement tout à fait originale. Dans quelle mesure celle-ci peut-elle se reproduire ? On traitera cette question de la reproduction de cette forme de développement en l'appréciant à deux niveaux :

- celui de sa reproduction sur place : problème de la continuité des systèmes localisés italiens;

- celui de sa reproduction en un autre endroit : problème de la reproductibilité des systèmes localisés italiens.

\subsection{La continulté des systèmes territoriaux italiens}

Les systèmes territoriaux italiens résultent d'un ensemble d'initiatives, de relations, de réseaux à tendances plus localisées et procèdent de l'entrée en scène d'acteurs spécifiques : petites entreprises, artisanat, secteur informel, etc. Mais ces démarches doivent répondre à un certain nombre d'exigences d'où la recherche de formes de régulation spécifiques. On peut dire que, jusqu'à une date récente, le marché et la «réciprocité» ont constitué les principaux mécanismes de régulation des systèmes territoriaux italiens.

Tous ces éléments qui ont été à la base du succès de ces zones d'industrialisation intense sont désormais confrontés à des mutations économiques et technologiques profondes en raison notamment de la concurrence internationale et sont soumis à un vaste processus de modernisation. Ces changements n'altèrent pas le modèle d'organisation économique et sociale des systèmes territoriaux mais rendent plus difficile sa reproduction élargie (Michelsons, 1987). Plusieurs aspects peuvent être rapidement évoqués : l'adaptation aux nouvelles technologies, la reproduction des compétences professionnelles et la reproduction entrepreneuriale.

\subsubsection{L'adaptation aux nouvelles technologies}

Tout d'abord l'accès d'une nébuleuse de petites et moyennes entreprises à des technologies de pointe peut s'avérer problématique. Leur taille les handicape, mais aussi la multiplicité des acteurs économiques participant à une même production. A cette difficulté s'ajoute le fait que nombre de districts industriels sont nés d'une utilisation intensive du travail, origine qui ne prédispose pas, a priori, à l'innovation technologique.

Pourtant, comme on l'a vu, la flexibilité productive assure une grande capacité d'adaptation. D'autre part, la caractéristique familiale de l'entreprise 
amplifie cette capacité d'adaptation : la décision de reconvertion ou d'innovations est plus rapide, ses effets, moins complexes que dans la grande organisation. Les alliances familiales structurent souvent des groupes d'entreprises, groupes apparents ou non, au sein desquels les expériences des uns et des autres se cumulent. Mais l'adoption de nouvelles technologies nécessite le dépassement de ces fondements traditionnels de la diffusion industrielle.

C'est ainsi que s'y ajoutent des initiatives plus récentes et plus originales (Ritaine, 1987). Dans la plupart des régions, l'esprit d'entreprise a gagné aussi le secteur du service aux entreprises resté longtemps moins dynamique. S'y sont lancés nombre de diplômés et d'experts (ingénieurs, techniciens ingénieurs, informaticiens, juriste, etc.) qui remplissent, pour les petites entreprises, les fonctions les plus délicates, dont celles de la recherche-développement et de l'expertise des nouvelles technologies. Un autre mouvement va dans le même sens : il s'agit de l'association d'entreprises sous la forme de coopératives ou de consortium capables de fonctionner très simplement et de respecter l'indépendance de chaque partenaire. Ces associations assurent les tâches les plus complexes, notamment : la recherche et l'évaluation des marchés potentiels, l'obtention de l'homologation des produits à l'extérieur, l'expertise de nouveaux types de machines, l'information sur la recherche et le développement de la recherche appliquée.

Reste que toute la recherche et l'expérimentation technologique ne peuvent être assumées ainsi; soit que les évolutions nécessaires concernent beaucoup plus d'acteurs que ne peut en ingérer un mouvement associatif, soit, au contraire, que la recherche ne concerne qu'un très petit nombre de spécialistes. Dans les deux cas, les investissements initiaux sont importants et requièrent l'intervention des pouvoirs publics; dans les systèmes industriels localisés italiens, il s'agit en général des régions ou des institutions municipales. Certaines de ces interventions se limitent à des actions de soutien financier (Toscane); d'autres aboutissent à la création de centres de services aux entreprises (Emilie-Romanie); certains de ces centres ont des vocations généralistes et d'information à tous les secteurs productifs; d'autres sont spécialisés, proposant une expertise précise à un secteur productif (centre de la céramique en Emilie-Romanie par exemple).

Ces expériences connaissent un développement plus ou moins heureux. Il apparaît cependant que ces interventions sont réelles et efficaces lorsqu'elles se situent en aval du milieu industriel, à sa demande et avec sa collaboration.

\subsubsection{La reproduction des compétences professionnelles}

Un autre point faible des systèmes territoriaux italiens est celui de l'enseignement professionnel. Les technologies nouvelles exigent un changement de qualification professionnelle à tous les niveaux : l'innovation du produit, l'innovation du processus de production, l'innovation dans la gestion et l'organisation des différentes fonctions des entreprises et du travail (Capecchi, 1987). La «socialisation 
familiale» au métier a beaucoup de mal à jour son rôle dans ce nouveau contexte. Les anciennes écoles professionnelles semblent également dépassées. Aussi, certaines régions - comme l'Emilie-Romanie - essaient de s'adapter en organisant des formations de deuxième niveau pour un travail salarié qualifié qui prend place entre l'ingénieur et l'ouvrier, ou des cycles de formation pour entrepreneurs et gestionnaires des petites entreprises (Capecchi, 1987). La formation professionnelle devient donc un point très important dans la reproduction des systèmes industriels localisés italiens et requiert de plus en plus l'appui des pouvoirs publics.

\subsubsection{La reproduction entrepreneuriale}

L'industrialisation a transformé la société des systèmes territoriaux bien audelà des changements morphologiques. Tout en réactualisant valeurs et pratiques d'un monde d'artisans, elle les a tirées vers celles d'un monde capitaliste : réussir, ce n'est pas seulement s' installer à son compte, c'est aussi réussir en termes capitalistes. Dans ce contexte, l'aspiration à l'ascension sociale est devenue l'un des ressorts principaux du système et souvent la catégorie d'entreprises s'est constituée par ouverture sociale. Mais passé la période de constitution du système local, les positions semblent se figer et les possibilités d'ascension sociale se restreignent. $\mathrm{La}$ maturité industrielle du système limite alors l'ouverture sociale alors que l'une des motivations principales des acteurs demeure cette ascension. Comme le montre très bien E. Ritaine à propos de Prato, la reproduction des systèmes devient alors un peu plus problématique (Ritaine, 1987).

Ainsi, les systèmes productifs territoriaux italiens doivent franchir un certain nombre de caps pour assurer leur reproduction à terme. Mais les fondements traditionnels du succès de ces zones ne suffisent plus. Le marché (entendu comme initiative individuelle et allocation spontanée des ressources) et la communauté (famille, alliances familiales, associations diverses) sont de moins en moins aptes à maîtriser les mutations en cours et à régulariser les rapports entre économie et société au sein même du système.

Les nouveaux besoins des systèmes territoriaux conjugués à une plus grande complexité de l'industrie et de la société se traduisent en demandes au système politique local. Celui-ci doit fournir les services nécessaires à la reproduction des systèmes territoriaux et doit «médiatiser» les intérêts constitués collectivement. Les pouvoirs publics locaux (régions, municipalités) doivent donc assurer un rôle important de régulation de plus en plus important. Dans ce mouvement, il ne s'agit pas de rejoindre le modèle d'organisation économique et social qui est celui des régions de la grande entreprise fordiste. Il s'agit plutôt d'amplifier les réseaux de collaboration plus ou moins informels qui existent déjà entre les entreprises de telle sorte que ceux-cis'affirment comme une véritable forme organisatrice intermédiaire entre l'organisation interne des systèmes et le marché. Il reste cependant que cette évolution «favorable» est loin d'être générale à tous les systèmes territoriaux italiens. 
On peut citer le cas de Vigevano dont l'industrie de la chaussure se restreint et où la société se fige et aspire à un monde bureaucratisé, sécurisé, sans risque. Il faut aussi s'interroger sur le caractère spécifique de ce redéploiement sur une base locale élargie au moment même où dans certains pays comme la France, c'est une mobilisation élargie des ressources extérieures qui est observée le long des réseaux de PME de plus en plus externes.

\subsection{La reproductibilité des systèmes productifs territoriaux dans les pays d'industrialisation récente}

Des systèmes productifs territoriaux se développent dans les pays d'industrialisation récente, constituant ainsi une composante non négligeable de leur croissance. En Espagne, ils se construisent dans la province de Valence, dans les plaines au Nord de Barcelone et dans la vallée du Vinalopo; au Portugal, ils se concentrent dans le Nord du pays dans les districts de Braga, Abveiro et Porto (Rui Silva, 1989); on rencontre aussi des systèmes productifs locaux à Fès au Maroc, à Ksar Hellal et Sfax en Tunisie; on peut aussi mentionner les aires d'industrialisation à base de PMI du pays Bamiléké au Cameroun et celle de l'île de Taïwan qui peuvent être considérées comme un véritable système productif à industrialisation diffuse. Ces systèmes émergent dans des conditions qui rappellent celles rencontrées dans le cas des districts industriels italiens.

\subsubsection{Importance des initiatives entrepreneuriales}

Une analyse de l'industrie de ces systèmes territoriaux renvoie à une problématique entrepreneuriale. Il s'agit de petites entreprises mues au départ avec un capital limité. On a affaire à des hommes qui sont propriétaires de leur entreprise et en assument le risque commercial, financier et technique. Autre caractéristique : l'imitation comme mode d'entrée dans l'industrie. Il y a une sorte de phénomène cumulatif s'accompagnant d'un nombre important de création de nouvelles entreprises dans le même métier, le même secteur.

\subsubsection{Une dynamique technologique «incrémentale»}

Dans l'ensemble, on est en présence d'une maîtrise technologique progressive. Très souvent en effet, l'évolution technique s'effectue à travers le passage du stade artisanal au stade industriel. A Sfax et à Fès par exemple, cette évolution s'accompagne de deux opérations conjointes : la modernisation de l'outil de production et le transfert des ateliers de la médina vers la ville nouvelle ou vers les quartiers industriels. Cette modernisation peut s'opérer par à coups en fonction de l'évolution du mode de vie et de la consommation donnant lieu à une «industrialisation composite» faisant que le métier soit en partie artisanal et industriel (Guerraoui 
et Fejjal, 1989). On est donc en présence d'une dynamique de type «incrémentale». Celle-ci entraîne une densification du tissu industriel local en donnant naissance à des opérations dites «d'intégration»: création d'une fonderie, d'ateliers d'outillage, etc.

\subsubsection{Un héritage pré-industriel réactualisé}

Le développement de ces systèmes repose en partie sur la possibilité de récupérer, en vue d'un développement moderne, toutes les forces et les ressources dont l'organisation économique et sociale pré-existante dispose. Dans bien des cas, il s'agit d'une industrialisation qui valorise et fait évoluer un artisanat ou une tradition manufacturière. A Sfax, la micro-entreprise et l'artisanat traditionnels sont à la base d'une dynamique rampante d'activités industrielles et d'innovations techniques (Bouchrara, 1985). A Fès, on note l'industrialisation des métiers de la tannerie qui se prolongent dans la chaussure ainsi que la modernisation et la mécanisation de l'habillement, du travail des métaux et du bois (Guerraoui et Fejjal, 1989). Au Cameroun, dans le pays Bamiléké, on observe également une telle évolution de l'artisanat vers l'industrie. Les systèmes locaux espagnols ou portugais apparaisssent également comme la valorisation et la modernisation de longues traditions manufacturières. Au total, il s'agit d'un héritage pré-industriel réactualisé évoluant vers l'industrie moderne.

\subsubsection{Milieux porteurs et autorégulations locales}

La dynamique des systèmes territoriaux dans les pays d'industrialisation récente procèdent également de phénomènes plus collectifs qui renvoient, par certains côtés, à la totalité de l'économie et de la société où se déroule le processus d'industrialisation. De ce point de vue, et comme en Italie, le milieu dans lequel $s$ 'amorce et se développe l'industrie joue un rôle fondamental.

A côté du marché, il faut souligner, tout d'abord, le rôle d'une solidarité territoriale organisée autour des métiers exercés localement. Il s'agit de rapports différents des relations courantes d'achats-ventes entre entreprises : ils n'ont pas forcément un caractère marchand et sont plus ou moins formalisés, mais ils sont à finalité productive. Ils portent sur des prêts de personnel et de machines, des coopérations occasionnelles, des discussions et des informations informelles. A Sfax, par exemple, des dizaines d'ateliers de la mécanique coopèrent et on observe des entreprises qui développent ensemble des prototypes et qui se concertent pour une utilisation plus rationnelle de leur capacité de production.

D'autre part, la dynamique industrielle est aussi fréquemment empreinte de la norme familiale. Dans le Sud du Portugal, l'industrialisation à partir de petites initiatives s'appuie sur une reproduction économique et sociale à dominante familiale et rurale; à Sfax, les entreprencurs sont issus du milieu local et ils créent en 
s'appuyant sur le réseau familial; à Taïwan, la plupart des PME sont familiales ou en association avec parents et amis.

Enfin, l'existence d'un système socio-culturel communautaire est un autre indice d'une réalité locale de suscitation de l'industrialisation à partir d'initiatives décentralisées. Dans le Nord du Portugal, la société repose sur une petite agriculture familiale qui est non seulement nécessaire à la reproduction de la force de travail industrielle, mais aussi au développement des initiatives entrepreneuriales (mobilisation de terrains, de biens fonciers pour créer l'entreprise par exemple). La société sfaxienne, de son côté, peut se définir comme un ensemble de communautés constituées autour d'activités rurales, commerciales et artisanales et autour de valeurs de références d'expressions culturelles de conduites économiques. $\mathrm{Au}$ Cameroun, l'organisation sociale très structurée, liée à l'agencement en concessions des cultures, le vif sentiment de solidarité, un système d'héritage exclusif au profit d'un fils privilégié, sont chez le peuple Bamiléké à l'origine du développement spontané de la petite entreprise. Enfin, l'homogénéité de la société, l'habitude de vivre en groupes structurés, la facilité des relations sociales, sont à la base du foisonnement industriel à Taïwan.

Au total, on est en présence d'un faisceau d'encadrements favorables (famille et ethnies, associations, villages, villes, etc.) expliquant largement la croissance en grappes l'une après l'autre de petites affaires, de petits ateliers et de petites et moyennes entreprises industrielles. Ces encadrements donnent lieu à une pratique, une régulation territoriale dont l'identité est finalement marquée et à un champ territorial dont l'autonomie est nettement affirmée. Identité et autonomie ne signifient pas pour autant isolement. Ces systèmes territoriaux démontrent également leur capacité à rebondir en fonction de sollicitations extérieures et se caractérisent par leur capacité à assimiler les influences extérieures pour leur propre développement.

\section{Conclusion}

Suite à cette analyse, on peut préciser la portée réelle de ce type de développement que constituent les districts industriels ou les systèmes productifs territoriaux.

Les systèmes territoriaux représentent un niveau d'organisation spatial spécifique qui n'est ni celui de l'entreprise comme entité isolable, ni celui des systèmes productifs nationaux découpés en branches, secteurs, filières et simplement projetés au niveau spatial. Il s'agit de systèmes qui se développent grâce à la capacité de certains territoires à valoriser le potentiel local. Cette «territorialité» est la résultante complexe d'identifications collectives, économiques, sociales, politiques 
et culturelles. Ces systèmes territoriaux naissent généralement dans des territoires marqués par une histoire riche de relations économiques et sociales entre acteurs.

Il s'agit d'une forme d'industrialisation bien adaptée aux exigences récentes de la flexibilité qui s'implante au sein de populations à prépondérance agricole et qui permet l'industrialisation de certains pays retardés. On serait en présence d'un modèle d'accession des sociétés traditionnelles à la modernité. Ce modèle repose sur la correspondance entre l'organisation économique et l'organisation sociale à un moment donné et sur un territoire particulier, correspondance que peut favoriser ou inhiber les politiques des Etats.

Il reste cependant la question du devenir de ce type de structure une fois parvenu à maturité : doit-elle céder la place à un modèle de développement concentré avec des grandes entreprises ou disparaître ? Une telle évolution serait du reste la simple réplique de ce qui s'est passé dans les pays les plus industrialisés. Mais avec la crise des grandes organisations et la recherche par celles-ci d'une plus grande flexibilité, il est probable qu'on s'oriente vers d'autres voies. D'une part, il apparaît que le développement des systèmes territoriaux sur une voie continue ne peut plus dépendre uniquement du jeu des automatismes car il suppose de plus en plus une prise de conscience claire et un dessein politique explicite. D'autre part, avec l'industrialisation croissante de l'économie et une mobilisation en réseaux des ressources qui s'effectue de plus en plus à l'échelle mondiale, il n'est pas certain que la reproduction élargie sur base locale soit l'itinéraire général; dans un certain nombre de cas, l'ancrage local ne constituerait alors que l'un des aspects de la réorganisation des systèmes productifs territoriaux. 


\section{BIBLIOGRAPHIE}

Aydalot, $\mathrm{Ph}$. , (1984), «A la recherche de nouveaux dynamismes spatiaux», dans Crise et Espace, Paris, Economica.

Bagnasco, A., (1985), «La contruzione sociale del mercato : strategie di impresa e esperimenti di scala in Italia», Stato e Mercato, $\mathrm{n}^{\circ} 13$.

Becattini, G., (1979), «Dal settore industriale al distretto industriale», Rivista de Economia e Politica Indistriale, $\mathrm{n}^{\circ} 1$.

Becattini, G., (1987), Mercato e forze locali : il distretto industriale, Bologne, Il Mulino.

Bertrand, O., (1986), «Ressources humaines et compétitivité de l'économie italienne», Rapport de mission CEREQ.

Bouchrara, M., (1985), «Industrialisation rampante et innovation clandestine en Tunisie», Cahiers IREP., D. $\mathrm{n}^{\circ} 9$.

Brutti, M., (1982), «La nuova centralità delle economie locale», Rassegna,Economica, $n^{\circ} 5$.

Capecchi, V., (1987), «Formation professionnelle et petite entreprise : le développement industriel à spécialisation flexible en Emilie Romagne», Formation et Emploi, $\mathrm{n}^{\circ} 19$.

Dewerpe, A., (1984), «Genèse proto-industrielle d'une région développée : l'Italie septentrionale (1800-1880)», Annales ESC, $\mathrm{n}^{\circ} 5$.

Fuà, G., (1985), «Les voies diverses du développement en Europe», Annales ESC, $\mathrm{n}^{\circ} 3$.

Garofoli, G., (1987), «Sviluppo regionale e ristrutturazione industriale : il modello italiano degli anni 70», Rassegna Economica, $\mathrm{n}^{\circ} 6$.

Guerraoui et Fejjal, (1989), «Artisanat et industrialisation à Fès», Histoires du développement, $\mathrm{n}^{\circ} 1$.

Michelsons, M., (1987), «La problemica dell'industrializzazione diffusa nelle scienze sociale italiani», dans Piccola città e piccola impresa, Milan, Franco Angeli.

Ritaine, E., (1987a), «Prato ou l'exaspération de la diffusion industrielle», Sociologie du travail, $\mathrm{n}^{\circ} 2$.

Ritaine, E., (1987b), «PMI et technologie en Italie : tous les moyens sont bons», Annales des mines, novembre.

Rui Silva, M., (1989), «Industrialisation et développement local : une interprétation à partie du cas portugais», Thèse de doctorat, Université des Sciences Sociales de Grenoble. 